\title{
RANDOM SEQUENTIAL PACKING OF CUBES
}


This page intentionally left blank 

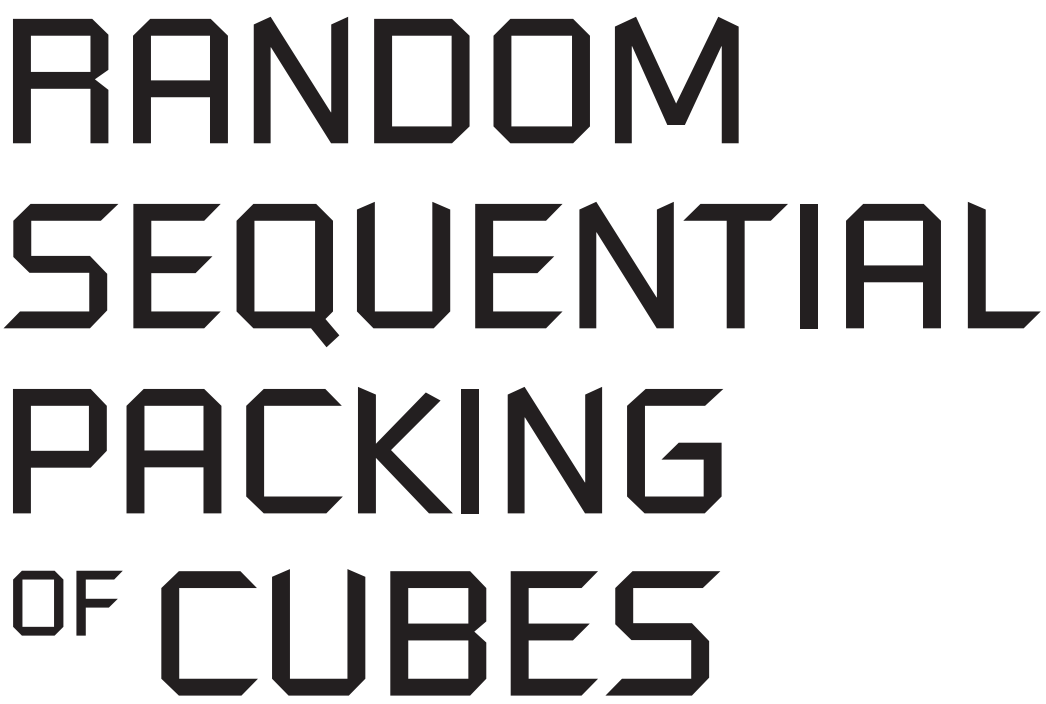

\section{Mathieu Dutour Sikirić}

Ruđer Bošković Institute, Croatia

\section{Yoshiaki Itoh}

The Graduate University for Advanced Studies, Japan

\& The Institute of Statistical Mathematics, Japan 


\title{
Published by
}

World Scientific Publishing Co. Pte. Ltd.

5 Toh Tuck Link, Singapore 596224

USA office: 27 Warren Street, Suite 401-402, Hackensack, NJ 07601

UK office: 57 Shelton Street, Covent Garden, London WC2H 9HE

\author{
Library of Congress Cataloging-in-Publication Data \\ Dutour Sikirić, Mathieu. \\ Random sequential packing of cubes / by Mathieu Dutour Sikirić \& Yoshiaki Itoh. \\ p. cm. \\ Includes bibliographical references. \\ ISBN-13: 978-981-4307-83-3 (hardcover : alk. paper) \\ ISBN-10: 981-4307-83-1 (hardcover : alk. paper) \\ 1. Combinatorial packing and covering. 2. Sphere packings. I. Itoh, Yoshiaki, 1943-
}

II. Title.

QA166.7.D88 2011

$511 \cdot .6--d c 22$

2010027617

\section{British Library Cataloguing-in-Publication Data}

A catalogue record for this book is available from the British Library.

Copyright (C) 2011 by World Scientific Publishing Co. Pte. Ltd.

All rights reserved. This book, or parts thereof, may not be reproduced in any form or by any means, electronic or mechanical, including photocopying, recording or any information storage and retrieval system now known or to be invented, without written permission from the Publisher.

For photocopying of material in this volume, please pay a copying fee through the Copyright Clearance Center, Inc., 222 Rosewood Drive, Danvers, MA 01923, USA. In this case permission to photocopy is not required from the publisher.

Printed in Singapore. 
To Maja and Yuri 
This page intentionally left blank 


\section{Preface}

A cube packing is a family of translates of the unit cube $[0,1]^{n}$ such that the intersection of any two of them has empty interior. If one starts from the empty set and add translates, chosen at random, of the unit cube sequentially until there is no space to pack any more, then one gets a nonextensible cube packing.

The Flory model is perhaps the first, such sequential random packing. It was introduced in Polymer Chemistry to model the behavior of catalyst and it considers integral translates of an interval $[0,2]$ inside another interval $[0, n]$ and asks for the limit packing density which represents then the efficiency of the catalyst. Another classical such model is the random parking problem, where drivers park their cars of length 1 at random in a parking place interval $[0, x]$. Such models have wide applications to statistical physics.

The first major result in the field is the computation of the limit density $C_{R}$ of the random parking problem by [Rényi (1958)]. [Palásti (1960)] conjectured that the average density for the sequential random packing of $[0,1]^{d}$ in $[0, x]^{d}$ converges to $C_{R}^{d}$ when $x$ goes to $\infty$. The existence of the limiting packing density was shown only recently by [Penrose (2001)] but the rigorous computation of the limit packing density is not known for dimension greater than 1 . To estimate this $d$-dimensional limit packing density, a classic method is the sampling procedure or Monte-Carlo simulation of the random sequential packing itself. The results of those simulations do not support Palásti's conjecture on the value of the limit packing density [Blaisdell and Solomon (1970)] and currently the limiting packing density is not known rigorously in dimension $d>1$.

The 1-dimensional random sequential packing problem can be solved within the framework of classical mathematical analysis. For example in 
order to study the recursion formula to get the limiting packing density by [Rényi (1958)], we introduce delay integral equations, for which we apply Laplace transform and then finally a Tauberian theorem which we prove completely. The minimum of gaps gives an interesting nonlinear delay integral equation [Itoh (1980)] whose analysis is amenable by tools of classical analysis which we introduce appropriately when needed. The alternative methodology of [Dvoretzky and Robbins (1964)] is also introduced as well as the related central limit theorem. We consider then a unified KakutaniRényi model, which interpolates between the Kakutani interval splitting and the Rényi sequential random packing [Komaki and Itoh (1992)]. We also consider a spin variant of the packing problem, where cars can park with spin-up or spin-down and compute the corresponding limit packing density [Itoh and Shepp (1999)]. By introducing the idea of interval tree, we can make a continuous model of binary search tree which is an important structure in Computer Science [Sibuya and Itoh (1987)]. The tree generated by the sequential bisection has less discrete structure than the original binary search tree and we can make an analogous analysis to the 1-dimensional random packing.

In general $d$-dimensional random cube packing problem are very hard to analyze mathematically for $d>1$. Thus simulations are of paramount importance for the understanding of the involved phenomena. We give the general tools for doing random sequential packing by introducing the covering problem, Voronoi diagrams and Delaunay tessellations, which have a lot of applications in Science and Technology. We explain how this works out for the examples of packing by spheres, cubes and cross polytope (unit ball for the Hamming distance in Coding Theory).

We consider the simplest random sequential packing with rigid boundary, i.e. a packing in which cubes of sidelength 2 are put sequentially at random into the cube of sidelength 4 , with a cubic grid of unit sidelength [Itoh and Ueda (1983)]. This simplest random sequential packing model seems to have some of the characteristics of the model by Palásti and to be related to the discrete geometry of cubes which has a long history of research going back to Minkowski. Computer Simulations up to dimension 11 , suggest that the packing density $\gamma_{d}$ satisfies $\gamma_{d} \simeq d^{-\alpha}$ with an appropriate constant $\alpha$, as in the case of random sequential coding by Hamming distance [Itoh and Solomon (1986)]. Despite being in many ways a much simpler model, this model is still essentially unsolved. However by using the special geometry of the face lattice of the cube, it is shown that the expected number of decrease of the packing density is less than $\left(\frac{4}{3}\right)^{d}$ at 
each step of the random sequential packing. This shows that the expected number of cubes at the saturation is larger than $\left(\frac{3}{2}\right)^{d}$ [Poyarkov (2005)]. We also consider an extension of this model where one packs cubes of size $N$ in a cube of side lengths $2 N$.

This packing led us to the introduction of the random sequential packing of cubes of sidelength 2 in a torus of sidelength 4 . Just like the previously considered rigid boundary model, this case allows us to introduce a combinatorial formalism to describe the corresponding cube packings. Thus we are able to apply the methods of exhaustive combinatorial enumeration to this setting. This allows us to find a number of remarkable combinatorial structures that could hardly have been found purely by hand computations. Then we consider a continuous variant of this model and we show that again we can describe it by a combinatorial model [Dutour Sikirić and Itoh (2010)]. This allows us to find some new remarkable structures and to find some extremal result on the number of parameters.

Whenever possible, we tried to give a description of the mathematical theory used when they are slightly non-standard and we give appendices on Complex Analysis, Laplace Transform, Renewal Theory, Space Groups and Exhaustive Combinatorial Enumeration. When several methods are possible to solve a problem, for example Tauberian Theorem and Complex Analysis, we have indicated both and discussed the relative merit of the possible approaches.

For discussion and suggestion on random sequential packing, we greatly thank J. E. Cohen, M. Deza, N. Dolbilin, M. Dutour Sikirić, J. M. Hammersley, T. Hattori, I. Higuti, F. Komaki, J. A. Morrison, Y. Nishiyama, A. Poyarkov, L. A. Shepp, R. Shimizu, M. Sibuya, H. Solomon, M. Tanemura, H. Tong and S. Ueda.

We thank also École Normale Supérieure of Paris, Institute of Statistical Mathematics, Hayama Center of the Graduate University for Advanced Studies, The Rockefeller University, National University of Ireland in Galway, Rudjer Bošković Institute of Zagreb, Technical University Delft, Mathematisches Forschungsinstitut Oberwolfach, Hausdorff Institute of Bonn for continued support. 
This page intentionally left blank 


\section{Contents}

Preface vii

1. Introduction 1

2. The Flory model 9

2.1 One-dimensional discrete random sequential packing . . . 9

2.2 Application of generating function . . . . . . . . . 10

2.3 Number of gaps . . . . . . . . . . . . . . . . . . . . . . . 13

2.4 Minimum of gaps . . . . . . . . . . . . . . 17

2.5 Packing on circle and numerical study . . . . . . . . 20

2.6 Appendix: Complex Analysis . . . . . . . . . . . . . 21

3. Random interval packing 23

3.1 The probabilistic setup of the problem . . . . . . . 24

3.2 The solution of the delay differential equation using Laplace transform . . . . . . . . . . . . 26

3.3 The computation of the limit . . . . . . . . . . 28

3.4 Packing on circle and the speed of convergence . . . . . 31

3.5 Appendix: The Laplace transform . . . . . . . . . . . 36

4. On the minimum of gaps generated by 1-dimensional random packing $\quad 39$

$4.1 \quad$ Main properties of $\operatorname{Pr}(L(x) \geq h) \ldots \ldots \ldots$

$4.2 \quad$ Laplace transform of $\operatorname{Pr}(L(x) \geq h) \ldots \ldots \ldots . \ldots . \ldots 42$

4.3 Numerical calculations for $a(h) \ldots \ldots \ldots \ldots$

4.4 Asymptotic analysis for $a(h) \ldots \ldots \ldots . \ldots 49$ 
4.4.1 Renewal equation technique ......... 50

4.4.2 Approximation for small $1-h \ldots \ldots . \ldots 53$

4.4.3 Approximation for small $h \ldots \ldots \ldots 6$

4.5 Maximum of gaps . . . . . . . . . . . . . 63

4.6 Appendix: Renewal equations . . . . . . . . . . . . . 64

5. Integral equation method for the 1-dimensional random packing 69

$5.1 \quad$ Estimating $M(x)-C_{R} x \ldots \ldots \ldots \ldots$

5.2 The variance and the central limit theorem . . . . . 76

6. Random sequential bisection and its associated binary tree 83

6.1 Random sequential bisection . . . . . . . . . . . 84

6.2 Binary search tree . . . . . . . . . . . . 86

6.3 Expected number of nodes at the $d$-th level $\ldots \ldots . . .991$

6.4 Exponential distribution and uniform distribution . . . 92

6.5 Asymptotic size of the associated tree . . . . . . . . . 93

6.6 Asymptotic shape of the associated tree . . . . . . . . . 94

6.7 More on the associated tree . . . . . . . . . . . . 97

7. The unified Kakutani Rényi model $\quad 99$

7.1 The limit random packing density . . . . . . . . . 100

7.2 Expectation and variance of number of cars for $l=0 \ldots 102$

7.3 The central limit theorem . . . . . . . . . . . . 106

7.4 Almost sure convergence results . . . . . . . . . . . . . 109

7.5 The limit distribution of a randomly chosen gap . . . . 112

8. Parking cars with spin but no length 123

8.1 Integral equations . . . . . . . . . . . . . . . . 124

8.2 Existence of the limit packing density . . . . . . . . 126

8.3 Laplace transform and explicitly solvable cases . . . . . 130

8.4 General solution methods . . . . . . . . . . . . . . 131

8.5 The power series solution . . . . . . . . . . 135

8.6 Numerical computations . . . . . . . . . . . . . . . . . . . 139

9. Random sequential packing simulations $\quad 145$

9.1 Sequential random packing and the covering problem . . . 145

9.2 Random packing of spheres . . . . . . . . . . . . . . 148

9.3 Random packing of cubes . . . . . . . . . . . . 151 
9.4 Random sequential coding by Hamming distance . . . . . 153

9.5 Frequency of getting Golay code by a random sequential packing . . . . . . . . . . . . . 157

10. Discrete cube packings in the cube 161

10.1 Setting of a goal . . . . . . . . . . . . . . 161

10.2 Reduction to another problem . . . . . . . . . . . 162

10.3 Proof of the theorem . . . . . . . . . . . . . 167

11. Discrete cube packings in the torus 171

11.1 Introduction . . . . . . . . . . . . . . . . 171

11.2 Algorithm for generating cube packings . . . . . . . . 173

11.3 Non-extensible cube packings . . . . . . . . . . . . . 176

11.4 The second moment . . . . . . . . . . . . . . 182

11.5 Appendix: Crystallographic groups . . . . . . . . . . 185

12. Continuous random cube packings in cube and torus 189

12.1 Introduction . . . . . . . . . . . . . . . . . . . . 189

12.2 Combinatorial cube packings . . . . . . . . . . . 191

12.3 Discrete random cube packings of the cube . . . . . . . 199

12.4 Combinatorial torus cube packings and lamination construction . . . . . . . . . . . . . 203

12.5 Properties of non-extensible cube packings . . . . . . . . 210

Appendix A Combinatorial Enumeration 219

A.1 The isomorphism and automorphism problems . . . . . 220

A.2 Sequential exhaustive enumeration . . . . . . . . . . . . 224

A.3 The homomorphism principle . . . . . . . . . 225

$\begin{array}{ll}\text { Bibliography } & 227\end{array}$

$\begin{array}{ll}\text { Index } & 237\end{array}$ 